\title{
Performance Comparison of Single-Pump FOPA and LRA in a 16-channel DWDM Transmission System
}

\author{
Sergejs Olonkins, Julija Putrina, Vjaceslavs Bobrovs \\ Institute of Telecommunication, Riga Technical University \\ Azenes St. 12-332, LV-1048 Riga, Latvia \\ sergejs.olonkins@rtu.lv
}

\begin{abstract}
Raman amplifiers and Fiber optical parametric amplifiers (FOPA) are positioned as the main future leading techniques for all-optical signal amplification. Distributed Raman amplifiers are known as the least noisy, but this type of amplifiers suffers from poor pumping efficiency at low input signal powers, therefore, discrete (lumped) Raman amplifiers (LRA) could be used instead. Both FOPAs and LRAs are the likeliest replacements of conventional EDFAs, but at this point it is not clear which of these two approaches is the most promising taking into account the quality of the amplified signal. Therefore, the goal of this article is to determine which of the two amplification techniques ensures less amplifier produced signal impairments in a 16-channel $10 \mathrm{Gbps}$ dense wavelength division multiplexed (DWDM) transmission system.
\end{abstract}

Index Terms-Fiber optical parametric amplifier; Discrete Raman amplifier; Wavelength division multiplexing; Interchannel crosstalk.

\section{INTRODUCTION}

During the last couple of decades, the demand for higher network capacity has been constantly increasing under the influence of all-time growing availability of broadband multimedia services and machine to machine applications. Cisco Visual Networking Index (VNI) forecast states that yearly global IP traffic will reach $3.3 \mathrm{ZB}$ by the end of 2021 [1]. This pushes research in fiber optical communications to seek for ways to increase transmission speeds and improving performance of existing transmission systems [2], [3].

Increasing the number of channels is the most common ways to extend the throughput of a wavelength division multiplexed (WDM) transmission system, as this does not require replacement of the expensive transmitter and receiver end hardware. Unfortunately, such approach uses broader wavelength band for transmission. The amplification band of conventional erbium-doped fiber amplifiers (EDFA), that are used in modern transmission systems, is highly limited (1530 nm-1562 nm), and at some point EDFAs will not be able to ensure gain over the whole wavelength band used for transmission in such systems [4], [5].

Manuscript received 28 March, 2018; accepted 20 September, 2018

This research has been supported by Riga Technical University, Institute of Telecommunications
This fact raises a need to find an alternative solution to conventional EDFAs for all-optical signal amplification. The most promising alternative to EDFAs are the two types of optical amplifiers that utilize fiber non-linearity for all optical signal amplification purposes: Raman amplifiers (RA) and fiber optical parametric amplifiers (FOPA). Distributed Raman amplifiers (DRA) are well known for their relatively broad gain spectrum, the shape of which can be changed by varying the parameters of the pumps, and for producing very little noise in comparison with conventional EDFAs [6]. Unfortunately, DRAs have one major drawback - poor pumping efficiency at low input signal powers. To increase amplification efficiency Raman amplifiers may be implemented using short spans of dispersion compensating fiber (DCF) or high non-linearity fiber (HNLF). Such amplifiers are called discrete (lumped) Raman amplifiers (LRA). This approach results in increasing of the amount of amplifier produced signal impairments in respect to DRAs [6]. Noise figure (NF) of $3 \mathrm{~dB}$ is achievable for LRAs, which is significantly less than the NF of a conventional EDFA (5 dB-6 dB) [6], [7].

FOPAs are mainly implemented using HNLFs, specially designed for parametric amplification. Same as RAs, FOPAs are capable of providing amplification over a significantly higher wavelength band than EDFAs: flat gain was achieved for over nearly $100 \mathrm{~nm}$ [8] in phase-insensitive mode and $170 \mathrm{~nm}$ in phase-sensitive mode [9] using a dual-pump FOPA. It is possible to achieve noise figures for FOPAs as low as $3 \mathrm{~dB}$ in phase-insensitive mode and in theory $0 \mathrm{~dB}$ NF in phase-sensitive mode [10].

Both FOPAs and LRAs are promising alternatives for replacement of conventional EDFAs, but at this point it is not clear which of these two approaches is the most promising taking into account the quality of the amplified signal. Therefore, the goal of this article is to determine which of the two amplification techniques ensures less amplifier produced signal impairments in a 16-channel $10 \mathrm{Gbps}$ dense wavelength division multiplexed (DWDM) transmission system. To make the comparison valid the performance of both amplifiers is compared using the same power of the input signal and the same amount of pump power. The preamplifier scenario was used to avoid the impact of four-wave mixing (FWM) produced inter channel 
crosstalk, that arises during transmission after amplification, on the quality of the signal, thus allowing to have a clear view on the amplifier produced signal impairments.

\section{Simulation SetuP}

In this section the simulation scheme that was used in this research is described. OptSim 5.3 simulation software was chosen for the simulations as this strong mathematical tool is known for ensuring high accuracy results for simulations of complex optical transmission system without requirements for high performance hardware. To reach the goal of this research a simulation scheme of a 16-channel $9.953 \mathrm{Gbps}$ (STM-64) DWDM transmission system with non-return-tozero intensity on-off keying (NRZ-OOK) modulation and 50 $\mathrm{GHz}$ channel spacing was introduced. This simulation scheme is displayed in Fig. 1.

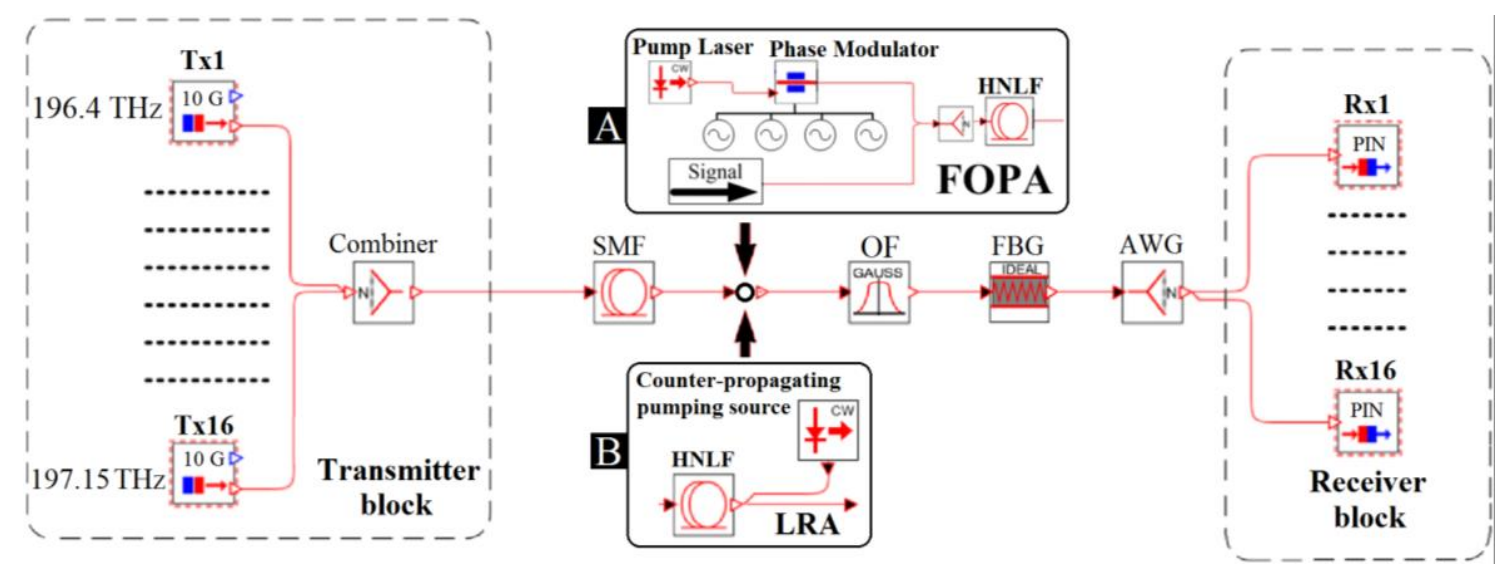

Fig. 1. Simulation model of 16-channel $10 \mathrm{Gbps}$ WDM transmission system with a FOPA (a) or an LRA (b) preamplifier for optical loss compensation.

As can be seen in Fig. 1, the simulation scheme consists of 3 main blocks - the transmitter block, the optical link and the receiver block. The transmitter block consists of 16 individual $10 \mathrm{Gbps}$ transmitters, each operating in frequency range from $196.4 \mathrm{THz}$ to $197.15 \mathrm{THz}$ with $50 \mathrm{GHz}$ channel spacing. Signal power of the signal at the output of each transmitter is equal to $1 \mathrm{~mW}(0 \mathrm{dBm})$. The optical radiation produced by all 16 transmitters is combined into a single optical flow using an optical combiner and sent through a $150 \mathrm{~km}$ long span of G.652.D standard single mode fiber (SMF). At the end of this fiber the signal is amplified by one of the two discrete amplifiers: the single pump FOPA (Fig. 1(a)) or the single pump LRA (Fig. 1(b)).

In the simulation model the amplifiers were placed at the end of the transmission link, and, therefore, were used as preamplifiers. Both amplifiers used a HNLF as the gain medium. The parameters of each HNLF are displayed in Table I. More detailed description of amplifier configuration is available in the following section.

TABLE I. HNLF PARAMETERS [11].
\begin{tabular}{|c|c|c|}
\hline Amplifier type & FOPA & LRA \\
\hline Attenuation at $\mathbf{1 5 5 0} \mathbf{~ n m} \mathbf{~ d B} / \mathbf{k m}$ & 0.96 & 0.62 \\
\hline Zero dispersion wavelength, $\mathbf{~ n m}$ & 1553.35 & 791.91 \\
\hline Non-linearity coefficient, $\mathbf{( W \cdot k m})^{-\mathbf{1}}$ & 15 & 15 \\
\hline Core effective area, $\boldsymbol{\mu m}^{\mathbf{2}}$ & 10 & 10 \\
\hline
\end{tabular}

After the signal was amplified it was sent through a bandpass optical filter with at least $3 \mathrm{~dB}$ insertion loss, with central frequency of $196.775 \mathrm{THz}$ and $2.5 \mathrm{THz}-3 \mathrm{~dB}$ twosided bandwidth. Afterwards, the signal was sent through a fiber Bragg grating (FBG) for dispersion compensation (with $3 \mathrm{~dB}$ insertion loss) and divided among 16 receivers using an arrayed waveguide grating (AWG) optical filter with $6 \mathrm{~dB}$ insertion loss. Each of the 16 receivers is based on a PIN photodetector with sensitivity of $-24 \mathrm{dBm}$ at reference biterror-ratio of $10^{-12}$.

\section{RESULTS AND DISCUSSION}

In this section the results obtained while configuring the FOPA and LRA preamplifiers in the previously described simulation model are introduced.

The process of parametric amplification is highly dependent on the phase mismatch between the interacting optical fields, thus, amplification efficiency of FOPAs dramatically decreases throughout the HNLF, along with accumulating dispersion caused linear phase mismatch between the pump and the signal. The performance of the LRA is not so highly dependent on the phase mismatch between the pump and the signal, and, therefore, significantly longer spans of HNLF can be used for the LRA. The higher the length of the HNLF for the LRA, the less pump power is needed to achieve a certain level of amplification. Unfortunately, at a certain point the amount of fiber non-linearity produced signal impairments becomes so high that it significantly degrades the quality of the amplified signal Therefore, the main idea of configuring the amplifiers was, at first, to find the lowest pump power that was capable of ensured BER values below the $10^{-12}$ threshold in all 16 channels in the system with the FOPA for a certain length of the HNLF fiber. Afterwards, the same pump power was used for the LRA to find the lowest HNLF length that could ensure the same condition for the quality of the amplified signal.

In order to find the lowest pump power that would ensure a certain level of amplification it is required to center the peak of the gain spectrum in respect to the spectrum of the amplified signal. This peak is being transferred towards higher frequencies along with increasing the pump power due to the non-linear part of the phase mismatch that also takes part in the process of parametric amplification. This is why while configuring the FOPA, both the power and the wavelength of the pump were changed simultaneously. The 
amplifier was based on a $1 \mathrm{~km}$ long HNLF the parameters of which are displayed in Table I. The obtained results have shown that the least FOPA pump power that ensured BER below the $10^{-12}$ threshold for all 16 channels was $590 \mathrm{~mW}$. The wavelength of the pump was set to $1553.9 \mathrm{~nm}$ (192.929 THz). The phase of the pump was modulated using the following 4 frequency tones in order to mitigate the unwanted impact of stimulated Brillouin scattering: $0.18 \mathrm{GHz}, 0.42 \mathrm{GHz}, 1.087 \mathrm{GHz}$ and $2.133 \mathrm{GHz}$. The gain spectrum that was provided by the FOPA with the above described configuration is displayed in Fig. 2.
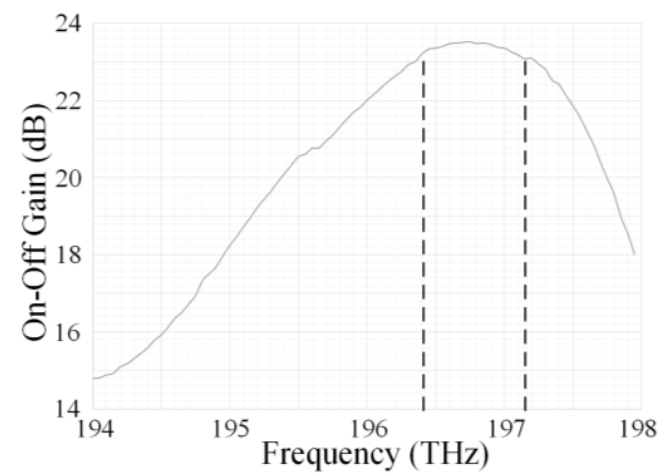

Fig. 2. Gain spectrum provided by the single-pump FOPA with $590 \mathrm{~mW}$ 192.929 $\mathrm{THz}$ pumping radiation.

As can be seen from Fig. 2, the peak of the gain spectrum coincides with the frequency of one of the center channels ( $8^{\text {th }}$ channel). The on-off gain among all 16 channels varied from $23.1 \mathrm{~dB}$ to $23.5 \mathrm{~dB}$ for signal with power level of $33.4 \mathrm{dBm}$ at the input of the amplifier.

For the LRA at first the wavelength of the pump was selected. Same as in the case of the FOPA the aim was to center the gain spectrum in respect to the 16 channels to be amplified. This can be achieved when the frequency of the pump is by $13.2 \mathrm{THz}$ higher than the center frequency of the transmitted signal: $209.975 \mathrm{THz}(1428.43 \mathrm{~nm})$. The same pump power as for the FOPA was used also for the LRA in order to find the shortest length of the HNLF that was capable of ensuing BER below the $10^{-12}$ threshold. The dependence of maximal BER value among all 16 channels on the length of the HNLF was observed. The obtained dependence is displayed in Fig. 3.

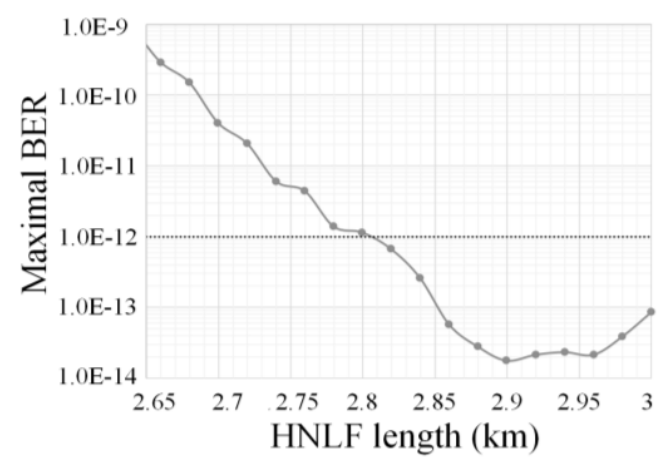

Fig. 3. Dependence of system maximal BER values on the length of the HNLF for the LRA with $590 \mathrm{~mW} 209.975 \mathrm{THz}$ pumping radiation.

As can be seen from Fig. 3 the shortest HNLF that could ensure BER below the $10^{-12}$ threshold in all the channels was $2.82 \mathrm{~km}$. Higher BER values at shorter lengths of HNLF is explained by insufficient amplification of the signal, but the increase for higher lengths of HNLF is associated with the increase of fiber nonlinearity produced signal impairments that, in its turn, causes significant decrease of amplified signal quality. The gain spectrum that was ensured by the LRA with $590 \mathrm{~mW} 209.975 \mathrm{THz}$ pumping radiation and $2.82 \mathrm{~km}$ long HNLF is shown in Fig. 4.

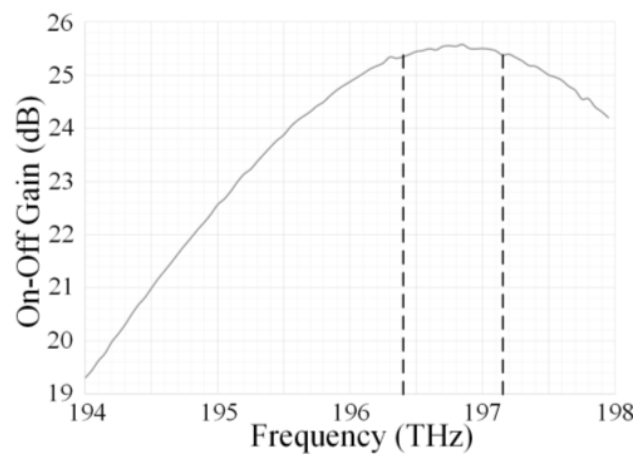

Fig. 4. Gain spectrum provided by the single-pump LRA with $590 \mathrm{~mW}$ 209.975 THz counter-propagating pumping radiation.

Figure 4 clearly shows that also in the case of the LRA the selected configuration on the pump has ensured gain the peak of which is centered in respect to the 16 channels of the amplified signal. The on-off gain ensured by the LRA varied from $25.3 \mathrm{~dB}$ to $25.6 \mathrm{~dB}$. In comparison with the FOPA the LRA required by approximately $2.1 \mathrm{~dB}$ more gain than the FOPA to ensure BER below $10^{-12}$ threshold in all channels. First of all, this is partially explained by the fact that the LRA used a 2.82 times longer HNLF, therefore additionally to the optical loss that accumulated during transmission the LRA provided on-off gain also needed to compensate higher amount of optical loss in the gain medium itself (HNLF). Secondly, this might indicate that in the case of the LRA the quality of the amplified signal is worse than in the system with the FOPA, therefore, the amplifier needs to provide additional gain to cover the BER increase caused by amplifier produced signal distortions.

To assess the quality of the amplified signal and to verify that the LRA produced signal impairments have actually caused the above mentioned need for additional amplification, the dependence of BER values on the power of the detected signal in the channel with the highest BER was obtained both in the system with the LRA and in the system with the FOPA. These results were compared to the same results obtained in an equivalent 16 channel DWDM transmission system, but without amplification, and power penalty values in respect to this system was obtained for both types of amplifiers. The obtained results are displayed in Fig. 5(a) for the system with the FOPA and in Fig. 5(b) for the system with the LRA.

In the system with the FOPA preamplifier the highest BER values were observed in the $14^{\text {th }}$ channel, where one of the lowest gain is observed. The fact that in the $15^{\text {th }}$ and $16^{\text {th }}$ channels BER values were lower even though the gain in the $14^{\text {th }}$ channel was slightly higher indicates, that inter-channel crosstalk is present in the system with the FOPA. In the system with the LRA the worst quality of the signal was actually observed in the channel with the highest level of amplification - this shows that the impact of fiber nonlinearity produced inter-channel crosstalk in this case is 
much higher than in the system with the parametric preamplifier.
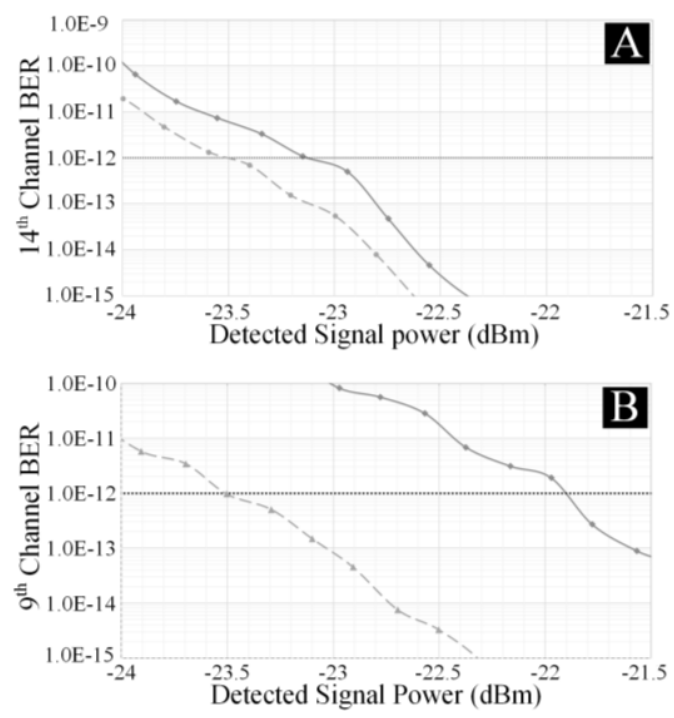

Fig. 5. Dependences of BER values on the power of the detected signal in the channels with worst quality of the signal of the system with the FOPA (a) and in the system with the LRA (b) preamplifiers (solid line) and in the corresponding channels of the system without amplification (dashed line)

This was confirmed by the results shown in Fig. 5. It was found that $0.35 \mathrm{~dB}$ power penalty exists between the system with the FOPA and the system without amplification (Fig. 5(a)), and $1.6 \mathrm{~dB}$ of power penalty between the system with the LRA and the system without amplification (Fig. 5(b)).

\section{CONCLUSIONS}

The goal of this research was to compare the performance of single-pump FOPAs and LRAs in exactly the same operating conditions, aiming to determine which of these two amplifier types generates less signal impairments. The comparison was made in a 16-channel 10 Gbps DWDM transmission system with FOPA and LRA preamplifiers.

First of all, such configuration of the FOPA was obtained that ensured BER values in all 16 channel below the $10^{-12}$ threshold using as low pump power as possible for a $1 \mathrm{~km}$ long HNLF: $590 \mathrm{~mW} 192.929 \mathrm{THz}$ pump was used. The same pump power was used while configuring the LRA to determine the shortest HNLF length that could ensure the required signal quality - a $2.82 \mathrm{~km}$ long HNLF was used for the LRA along with the $590 \mathrm{~mW} 209.975 \mathrm{THz}$ pump.

The above mentioned configuration of the FOPA has ensured from $23.1 \mathrm{~dB}$ till $23.5 \mathrm{~dB}$ of on-off gain for the 16 channels, which was by approximately $2.1 \mathrm{~dB}$ less than the LRA with the previously described parameters. Such difference in the level of amplification, required to ensure the same BER values, is explained in the following way:

1. The LRA used 2.82 times longer span of HNLF, therefore, additionally to the optical loss that accumulated during transmission the LRA provided on-off gain also needed to compensate higher amount of optical loss in the HNLF;

2. Because the LRA ensured less gain difference among all channels that the FOPA $(0.3 \mathrm{~dB}$ and $0.4 \mathrm{~dB}$ respectively), the difference in the required level of amplification clearly indicated that the signal at the output of the LRA was more distorted than at the output of the FOPA.

The $2^{\text {nd }}$ assumption was also confirmed when the BER value dependences on the power of the detected signal were obtained for the channels with the highest BER and compared to the corresponding dependences obtained in a system without amplification: $0.35 \mathrm{~dB}$ of power penalty was observed in case when the FOPA was used, but for the LRA this power penalty reached $1.6 \mathrm{~dB}$.

Such difference in power penalty is explained by the fact that the LRA produced amplification was less efficient than parametric amplification, therefore longer span of the HNLF was required for the LRA to ensure the same level of amplification. Because of this, in the case of the LRA, fiber non-linearity produced signal impairment (especially FWM produced inter channel crosstalk) were accumulated over a longer span of the HNLF. Furthermore, as it can be concluded from the results displayed in Fig. 3, the more gain the signal has obtained, the more significant arising of fiber non-linearity took place, and the more the amplified signal got distorted. In this case also using less pump power would not solve the problem, as then a longer span of HNLF would be required to achieve the required gain.

\section{REFERENCES}

[1] Cisco Inc., "Cisco Visual Networking Index: Forecast and Methodology, 2016-2021”, White paper, 2016.

[2] S. K. Routray, A. Javali, R. Nyamangoudar, L. Sharma, "Latching on to Keck's law: Maintaining the high speed trends in optical communication", 4th Int. Conf. Advanced Computing and Communication Systems (ICACCS 2017), Coimbatore, 2017, pp. 15. DOI: 10.1109/ICACCS.2017.8014637.

[3] R. B. Patel, D. K. Kothari, "Performance enhancement of $12 \times 160$ Gbps (1.92 Tbps) WDM optical system for transmission distance upto $8000 \mathrm{~km}$ with differential coding", 5th Nirma University Int. Conf. Engineering (NUiCONE 2015), Ahmedabad, 2015, pp. 1-6, DOI: 10.1109/NUICONE.2015.7449623.

[4] M. H. Al-Mansoori, A. S. Al-Qasmi, K. M. Al-Abri, W. S. Al-Ghaithi M. A. A. Younis, "Wideband EDFA utilizing short-length high concentration erbium-doped fiber", IEEE 5th Int. Conf. Photonics (ICP 2014), Kuala Lumpur, 2014, pp. 201-203. DOI: 10.1109/ICP.2014.7002355.

[5] M. N. Islam, Raman Amplifiers for Telecommunications. Springer, USA, 2004, p. 298. DOI: 10.1109/JSTQE.2002.1016358.

[6] C. R. S. Fludger, V. Handerek, R. J. Mears, "Fundamental noise limits in broadband Raman amplifiers", IEEE Optical Fiber Communication Conf. and Exhibit. Technical Digest Postconf. Edition (OFC 2001), Anaheim, CA, 2001. DOI: 10.1109/OFC.2001.927820.

[7] J. D. Marconi, J. M. Chavez Boggio, H. L. Fragnito, "Nearly 100 nm bandwidth of flat gain with a double-pumped fiber optic parametric amplifier", in Proc. OFC 2007, 2007. DOI: 10.1109/OFC.2007.4348839.

[8] R. Malik, A. Kumpera, M. Karlsson, P. Andrekson, "Demonstration of ultra wideband phase-sensitive fiber optical parametric amplifier", IEEE Photonics Technology Letters, vol. 28, no. 2, pp. 175-177, 2016. DOI: 10.1109/LPT.2015.2488102.

[9] Hansryd, P. Andrekson, M. Westlund, Jie Li, P. Hedekvist, "Fiberbased optical parametric amplifiers and their applications", IEEE Journal of Selected Topics in Quantum Electronics, vol. 8, no. 3, pp. 506-520, 2002. DOI: 10.1109/JSTQE.2002.1016354.

[10] D. Chen, B. Sun, "Multi-wavelength fiber optical parametric oscillator based on a highly nonlinear fiber and a Sagnac loop filter", Progress in Electromagnetics Research, vol. 106, pp. 163-176, 2010. DOI: 10.1109/CLEOE-EQEC.2017.8086857.

[11] T. Okuno, M. Hirano, T. Nakanishi, M. Onishi, "Highly-nonlinear optical fibers and their applications", SEI technical review, Sumitomo electric Industries, vol. 62, pp. 35-39, 2006. 